\title{
LA MESCOLANZA LINGUISTICA COME FORMA DI COMUNICAZIONE ESPRESSIVA: IL CASO DEL CANTO POPOLARE BERGAMASCO
}

Mattia Sonzogni ${ }^{1}$

\section{INTRODUZIONE}

Il canto popolare rappresenta una forma espressiva altamente formalizzata che combina la dimensione testuale delle parole con quella musicale della melodia. Esso è soggetto tanto a istanze metriche quanto melodiche. Il risultato estetico deriva da un'azione combinata di musica e testo che si presentano alla fruizione come un unico inscindibile oggetto.

Lo studio del canto popolare in area italiana pone alcuni problemi connessi a due aspetti fondamentali che distinguono la musica popolare da altre manifestazioni sonore prodotte dalla cultura occidentale in età moderna e contemporanea: l'oralità ${ }^{2}$ (i canti conoscono una fruizione e una trasmissione essenzialmente orale) e l'ampia diffusione territoriale e sociale.

Concentrando l'attenzione sul secondo termine di questo binomio, viene riconosciuto dalla maggior parte degli etnomusicologi, e non solo, come i confini linguistici non costituiscano una discriminante sufficiente nell'individuare delle linee di cesura fra tipi di repertori diversi per conformazione e stile. Questo non è solo vero in senso diatopico, in quanto è difficile definire per quali elementi si differenzi, ad esempio, il repertorio bergamasco da quello bresciano o milanese, ricorrendo esclusivamente alle differenze dialettali, ma è valido anche in senso diastratico e diafasico. Infatti in area italiana la

${ }^{1}$ Università degli Studi di Milano.

${ }^{2}$ Sul problema dell'oralità si vedano Ong, 1987; Saussy, 2016; Neziri-Scaldaferri, 2016. Importante è anche il filone di ricerca rappresentato dai performance studies. 
testualità popolare realizza una vera e propria mescolanza linguistica, che, come vuole dimostrare il presente articolo, ha funzioni perlopiù espressive. A trovarsi impiegati nei testi popolari sono tanto elementi della lingua italiana quanto ampie tessere dialettali. L’ibridismo linguistico è una caratteristica fondamentale di questo tipo di repertorio, tanto da sopravvivere ancora oggi in una fase di avanzata alfabetizzazione degli abitanti della penisola. Questa doppia faccia linguistica è dovuta alla situazione di sostanziale diglossia in cui si muovevano i performers che hanno dato vita, o danno tutt'ora vita ${ }^{3}$, al repertorio popolare. Da una parte il dialetto, la lingua della quotidianità e della comunicazione orale, dall'altra l'italiano, la lingua ufficiale e pubblica, tipica della comunicazione scritta ${ }^{4}$. La natura ibrida della lingua dei canti popolari riflette quindi la situazione di diglossia che ha contraddistinto gran parte della popolazione italiana fra Ottocento e Novecento.

La mescolanza linguistica realizzata fra italiano e dialetto non rispecchia però solamente la situazione socio-culturale dell'Italia unita ma obbedisce anche a ragioni di natura espressiva, estetica e comunicativa.

Il presente articolo prende quindi in esame i modi e le finalità di tale miscela. Seppur il fenomeno sia comune a tutta la penisola, come caso di studio è stato scelto il repertorio popolare bergamasco, per dar meglio conto delle grandi escursioni e varietà linguistiche presenti in un territorio relativamente circoscritto nel catalogo di un gruppo definito e limitato di performer. Come fonti sono state utilizzate alcune raccolte ${ }^{5}$ realizzate dal secondo dopoguerra in avanti che si distinguono per la loro accuratezza scientifica e per la quantità e varietà di materiale presentato.

In un primo momento vengono definite le modalità di tale mescolanza affrontando un'analisi dei diversi codici che si trovano frammisti ed impiegati nel canto popolare. Punto di riferimento per questa prima parte è la definizione di lingua mista data da

\footnotetext{
${ }^{3} \mathrm{La}$ situazione linguistica presa in considerazione in questo passaggio e di conseguenza nell'intero articolo si riferisce perlopiù al passato (dalla metà dell'Ottocento fino agli ultimi decenni del Novecento). Tuttavia alcune distinzioni e considerazioni rimangono valide per la situazione presente dove permangono, presso una fascia della popolazione più anziana e nei gruppi sociali meno esposti alla scolarizzazione e isolati rispetto ai centri urbani, fenomeni di diglossia e di bilinguismo sociale.

${ }^{4}$ Per un'originale riflessione sociolinguistica e letteraria sul bilinguismo delle classi popolari si vedano le opere di Luigi Meneghello.

5 Anesa-Rondi, 1978; raccolta relativa al paese di Parre (BG) e Biella-Zani (1995) dove vengono riportati i numerosi canti appartenenti al repertorio degli Zani, storica famiglia di Dossena (BG).
} 
Glauco Sanga in un suo fondamentale studio ${ }^{6}$. Tale definizione viene discussa, rimodulata ed approfondita in una più ampia tassonomia che considera l'ampia differenza di registri linguistici e non la mescolanza meccanica fra il codice dialettale e la lingua ufficiale. Le considerazioni svolte partono concentrando il fuoco su elementi di carattere prettamente linguistico per poi ampliarlo e proporre una tassonomia che tenga conto anche di fattori stilistici e retorici.

Successivamente, superando la dicotomia lingua-dialetto, vengono considerati canti che presentano tessere alloglotte, estranee sia al dialetto locale che alla lingua ufficiale. Assieme all'alloglossia sono presi in esame $\mathrm{i}$ fenomeni di riflessione linguistica (metalinguismo). Tramite questi esempi è possibile mostrare come la manipolazione linguistica sia un fatto sostanzialmente creativo ed espressivo, le cui logiche divengono puramente estetiche, trascendendo dunque fatti di pura natura sociolinguistica, pur avendo alla loro base una forte motivazione antropologica. La mescolanza linguistica realizza delle precise finalità espressive e comunicative.

Il presente contributo dunque si concentra maggiormente sugli aspetti linguistici della musica folclorica, ma non va dimenticato che lingua e musica sono, per questo fenomeno, fuse ed inscindibili. L'analisi offerta nelle seguenti pagine sulla testualità popolare è quindi frutto di una netta scelta di campo, separando ciò che nasce inseparabile e che tale rimane nelle voci e nelle orecchie dei performer.

\section{REGISTRI LINGUISTICI}

Glauco Sanga ${ }^{7}$, nell'analizzare la lingua dei canti raccolti a Cigole (BS), classifica i testi in tre categorie: canti in dialetto, canti in lingua mista e canti in italiano.

Sanga nota come il dialetto non sia affatto dominante ma costituisca una componente minoritaria limitata ad alcuni generi e funzioni. L'italiano è presente perlopiù nella variante regionale ed è riscontrabile in canti di origine recente, riferiti alla prima guerra mondiale e ad altri fatti storici del primo Novecento. La lingua mista italiano-dialetto invece si presenta come un elaborato codice poetico-letterario che accosta dialetto ed italiano secondo ragioni prevalentemente metriche. La natura estetica di questa ibridazione linguistica giustifica Sanga nell'attribuirle il nome di lingua epico-lirica in

\footnotetext{
${ }^{6}$ Sanga, 1984.

${ }^{7}$ Sanga, 1984: 225 - 244.
} 
quanto tipica di generi come la ballata di evidente e spiccata derivazione letteraria e colta.

Nel prendere in esame la lingua dei canti popolari in territorio bergamasco intendiamo discostarci dalla suddivisione proposta da Sanga nei suoi lavori.

Il parlante bergamasco medio si muoveva nell'Ottocento in una condizione di sostanziale diglossia: da una parte la lingua ufficiale scritta ${ }^{8}$, l'italiano, dall'altra il dialetto che domina il campo dell'espressione orale. Questa situazione si riflette anche sulla testualità popolare che risulta divisa fra due poli di attrazione, il dialetto e la lingua. La lingua dei testi è in gran parte un sistema ibrido in cui è difficile ravvisare degli esempi dove uno dei due poli linguistici sia utilizzato in modo puro senza contaminazioni di sorta. Prevale invece il mescolamento di materiali linguistici eterogenei. A volte la natura metricamente equivalente della forma dialettale e italiana per lo stesso significato facilita la volatilità della conformazione linguistica di uno stesso canto, in talune esecuzioni uniformandolo alla norma italiana mentre in altre mantenendo una forte identità dialettale.

Italiano e dialetto non sono quindi due sistemi opposti in cui si concreta il canto popolare ma si presentano come due situazioni limite che danno luogo a sfumature via via meno accentuate in direzione di una lingua mista che è punto d'incontro in questa tassonomia (più che una categoria a sé stante). Le due categorie si declinano in una serie di registri, in cui rimangono valide le osservazioni circa i metri e i generi svolte da Sanga, che tendono da una parte al purismo mentre dall'altra ammettono senza problemi l'ibridazione linguistica. Pertanto, invece che considerare italiano e dialetto come categorie discrete, vengono identificati diversi registri di italiano e registri di dialetto nella consapevolezza della difficoltà di classificazione di ciascun testo. Infatti, nel determinare la forma linguistica di un testo è determinante la competenza linguistica del performer che

\footnotetext{
${ }^{8}$ Le raccolte di canti popolari prese in esame per il presente studio (Boninelli, 2010; Bernani, 1985; Bermani-Boninelli, 2001 sono edizioni critiche di raccolte di eruditi ottocenteschi mentre Anesa-Rondi, 1978; Biella-Zani, 1995 sono due ricerche monografiche condotte secondo il metodo etnomusicologico) sono tutte posteriori al periodo risorgimentale, momento in cui si afferma su gran parte della penisola un modello linguistico duplice. Da una parte la lingua scritta, che si impara a scuola e che viene impiegata negli atti pubblici e ufficiali, spesso di difficile comprensione e lontana dalla realtà quotidiana. Dall'altra il dialetto, la vera lingua materna che si impara in tenera età. Il lento ma inesorabile processo di italianizzazione della penisola si riflette dunque anche nel canto popolare, soprattutto confrontando le raccolte ottocentesche (Bermani, 1985; Boninelli, 2010; Bermani-Boninelli, 2001) con quelle più recenti (Anesa-Rondi, 1978; Biella-Zani, 1995).
} 
dà vita al canto attraverso un'azione di ri-creazione continua della veste linguistica di un testo.

I registri sono individuati considerando tanto istanze linguistiche quanto stilistiche. Italiano e dialetto sono stati suddivisi su tre livelli di registro: misto, dove è massimo il grado di mescolanza fra i due codici; a predominante, dove domina uno dei due codici con debole emersione di elementi dell'altro; puro dove l'influenza di elementi linguistici esterni è ridotta all'osso o addirittura assente. La denominazione del registro è inoltre affiancata da un'indicazione stilistica. Come messo in evidenza dalle classificazioni tematico-funzionali proposte da Roberto Leydi ${ }^{9}$ e dalla tassonomia linguistica rilevata da Sanga nei canti di Cigole, metro, lingua e argomento appaiono intrecciati profondamente. Proporremo denominazioni relative all'aspetto stilistico-tematico dei testi riconoscendo come determinate realizzazioni verbali e strutture linguistiche siano caratteristiche privilegiate di alcune forme rispetto ad altre. Rimane tuttavia impossibile porre in relazione univoca lingua e stile, come invece avviene nella letteratura colta fino ai primi dell'Ottocento.

La pluralità testuale del canto popolare non ne favorisce la classificazione tramite categorie discrete. Tuttavia è evidente l'esistenza di alcuni meccanismi di costruzione e di uso linguistico che, se non maggioritari, rimangono in ogni caso predominanti nello sviluppo del repertorio. Nell'esplorare le varie gradazioni linguistiche che vanno dalla lingua al dialetto analizzeremo canti provenienti da un'unica raccolta, quella di Parre ${ }^{10}$, per testimoniare maggiormente l'estrema varietà di soluzioni riscontrabili all'interno di un unico territorio fra un numero ridotto di parlanti.

Sia per quanto riguarda la lingua che per quanto riguarda il dialetto l'analisi muove dai registri più vicini alla definizione di lingua mista proposta da Sanga verso forme linguisticamente meno ibride.

\subsection{Registri di italiano}

All'interno dei registri di italiano distinguiamo tre livelli: un registro misto o narrativo, con mescolanza di elementi dialettali e italiani secondo ragioni metriche e testuali; un registro a predominante o drammatico dove l'italiano appare profondamente condizionato dal

\footnotetext{
${ }^{9}$ Leydi, 1972.

10 Anesa Rondi, 1978.
} 
sostrato regionale; un registro puro o ufficiale in cui troviamo una compiuta padronanza linguistica con rarissime emersioni del locale.

\section{a) registro misto: narrativo}

Questo registro linguistico è tipico della forma metrico-musicale della ballata. Il testo della ballata si caratterizza per la dimensione spiccatamente narrativa che concentra in uno spazio relativamente breve il racconto di un episodio narratologicamente compiuto. La forma testuale presenta un alto tasso di formalizzazione: si articola secondo un preciso schema metrico, comportando l'adozione di un linguaggio costruito artificialmente e letterariamente rispetto allo stile della conversazione quotidiana. La natura artificiosa di questo registro sta nella commistione continua di termini dialettali con voci della lingua italiana. Le forme non sono semplicemente contrapposte, rileva Sanga, ma costituiscono un organismo testuale simbiotico. La scelta di parole dialettali o italiane non avviene secondo la libera volontà del parlante ma si verifica in obbedienza a precise regole metriche, collocando i termini di una o l'altra varietà in sedi obbligate.

Considerando il testo della ballata l'è la figlia d'un pastore è possibile notare che il verso è diviso in due emistichi. Nella performance il primo viene ripetuto una volta mentre dopo la prima comparsa intera del verso esso è ripetuto una seconda volta ${ }^{11}$.

$$
\begin{aligned}
& \text { l'è la figlia d'un pastór töc i dis che lé l'è bèla } \\
& \text { la farémo rimiràr dai soldati che fan la guèra } \\
& \text { e 'lpö bèl che l'gh'éra dèt l'è stac chèl che l'l'à rubada } \\
& \text { e l'l'à portada di lontàn in un castèllo oscuro oscuro } \\
& \text { e l'l' à lasada là sèt agn sènsa mai vedér la luna } \\
& \text { e pasàt fò chèl di sèt agn a l'ghà apèrto öna finestrèlla } \\
& \text { e la guardava in mèśso al mar che passava il bastiménto } \\
& \text { e l'gh'era dént ol sò papà che l'tornava dalla Francia } \\
& \text { o papà caro papà cóśa dìghel de mé la Francia }
\end{aligned}
$$

11 Per rendere più agile la lettura del testo a fini analitici riportiamo ciascun verso senza le ripetizioni. Con le ripetizioni le strofe si presentano secondo questo schema:

L'è la figlia d'un pastore

L'è la figlia d'un pastor töc $i$ dis che lè l'è bèla

L'è la figlia d'un pastor töc $i$ dis che lè l'è bèla 
töc $i$ dis che té to sé la mia figlia derubada

e l'anelìn che gh'ó sö'l dit a l'è 'l sègn che só spuśada

e la gh'avéva un fanciullìn che maneggiava già la spada. ${ }^{12}$

Linguisticamente il testo alterna, secondo un rapporto di quantità equivalente, dialetto e italiano. Siamo nell'ambito della lingua mista identificata da Sanga. L'uso del dialetto o dell'italiano rispetta alcune regole e criteri strutturali.

Il verso è divisibile in due emistichi di cui il primo è ossitono mentre il secondo è parossitono. Il dialetto bergamasco è ricco di parole ossitone mentre è povero di termini parossitoni che abbondano invece nell'italiano. Così nel finale del primo emistichio riscontriamo una maggiore prevalenza di voci dialettali (dèt, agn, sé, dit) che nel finale del secondo emistichio, dove sono più frequenti i termini in lingua (oscuro, luna, finestrèlla, bastiménto, Francia, spada). Possibile e frequente è anche il caso contrario, là dove le forme sono foneticamente molto vicine e simili alle loro rispettive varianti in italiano o dialetto.

Accanto a queste posizioni obbligate troviamo principi di uniformazione linguistica su base sintattica e testuale. Ad esempio notiamo che i due discorsi diretti liberi presenti (al verso 2 e ai versi 10 - 11) sono uniformi dal punto di vista linguistico. Il primo è in italiano, il secondo, uno scambio privato e personale fra padre e figlia, in dialetto, la lingua madre che l'ascoltatore suppone che i due personaggi parlino. La scelta di un codice linguistico a discapito dell'altro avviene quindi non solo per ragioni metriche ma anche per fini espressivi e stilistici.

Per quanto riguarda l'italiano, notiamo che è realizzato secondo un registro a tratti debitore del parlato dialettale e della varietà regionale: l'è la figlia con la ripetizione del pronome atono tipica del dialetto; guèra presenta lo scempiamento della $r$, mèsśso (anziché mezzo) presenta il passaggio dall'affricata dentale alla fricativa alveolare, tipico del sistema fonologico lombardo per cui $[\mathrm{ts}]>[\mathrm{s}]$ e $[\mathrm{dz}]>[\mathrm{z}]^{13}$. Dall'altro lato notiamo anche un uso dell'italiano che risente di modelli letterari e paraletterari, come è evidente dall'impiego di voci quali rimirar, castello, oscuro, fanciullin.

12 Anesa-Rondi (1978), pp. 119 - 121. Traduzione: [ . . . . . / tutti dicono che lei è bella / . . / e il più bello fra quelli / ... è stato quello che l'ha rubata $/ \ldots / \ldots / \ldots /$ l'ha lasciata là sette anni $/ \ldots /$ passato il settimo anno / . . / le ha aperto una finestrella / .. / . . c'era dentro suo papà / . . / . / cosa dice di me la Francia / . . / tutti dicono che tu sei / .. / la mia figlia rubata / . . / l'anellino che ho sul dito / ... / è il segno che sono sposata / ...].

13 Sanga, 1984: 18. 
Il dialetto è padroneggiato con sicurezza anche se si nota una leggera erosione a favore dellitaliano (ad esempio lontan al posto di luntà alla fine del primo emistichio del quarto verso, in una sede, come visto, privilegiata per i termini dialettali).

Sanga definisce questo impasto linguistico una lingua dal carattere letterario in quanto l'unione di forme linguistiche italiane e dialettali è costitutiva della forma metricotestuale della ballata. È quindi da escludere un'origine dialettale con un successivo passaggio verso l'italiano e viceversa. D'altro canto è innegabile come la vicinanza fonetica di alcune forme dialettali con le equivalenti italiane (per esempio lontan e luntà) favorisca un maggior grado di mutevolezza linguistica in dipendenza dall'arbitrarietà del performer.

La costruzione sintattica è elementare e brachilogica. Prevale l'uso anaforico della congiunzione coordinante $e$ all'inizio del verso. La subordinazione è realizzata esclusivamente attraverso l'uso della congiunzione che a introdurre subordinate oggettive e relative. Notiamo inoltre l'uso del che polivalente: e la guardava in mèsśo al mar che passava il bastimento. Tali tratti linguistici accentuano il carattere narrativo del testo, che delinea con semplicità una concatenazione di fatti.

\section{b) registro a predominante: drammatico}

All'interno del registro drammatico inseriamo testi che presentano una padronanza più sicura dell'italiano. La norma linguistica è osservata con maggiore precisione ed è maggiormente vicina allo standard, rimanendone però lontana a causa del frequente affiorare di tratti tipici del parlato con influssi di varianti regionali.

In questa categoria possono essere classificati testi di origine più recente rispetto alle ballate, che invece presentano una storia più lunga e complessa, inserendosi in un contesto non solo nazionale ma panromanzo ed europeo ${ }^{14}$. Si tratta di testi formatisi nel corso dell'Ottocento grazie all'apporto di autori con una maggiore competenza nell'ambito della letteratura colta. Anche in questo caso l'impronta narrativa è forte ma è evidente una maggiore incidenza del patetismo tipico del mondo dell'opera lirica. I fatti vengono caricati di una forte emotività e drammaticità.

Come esempio riportiamo il canto Ferdinando s'innamora.

${ }^{14}$ Sulle relazioni fra ballate di aree territoriali diverse, a livello italiano ed europeo, si veda Dell'Armellina, 2008; Saffioti, 2008. 
1 Ferdinando s'innamóra

di una bèlla, di una bèlla ragassina

che di nóme erà Lisêtta

éra nata, éra nata per l'amór

\section{Di frequènte la portava}

giù nei bóschi, giù nei bóschi fóschi fóschi

finchè vinse al sé l'onóre

e poì dòpo, e pòi dòpo la lasciò

3 La sua śia se ne acòrse

córse a caśa, córse a caśa da Lisètta:

- dimmi un po' cara Lisètta

sèi scambiata, sèi scambiata nei colór

\author{
4 La matina, il ciél seréno \\ Ferdinando, Ferdinando s'incammina \\ con Lisêtta per un braccio \\ và alla chiéśa, và alla chiéśa per riposar \\ 5 Quan' fu stati all'altare \\ sènsa fare, sènsa dire una paròla \\ con un cólpo di rivolteilla \\ mòrta a tèrra, mòrta a tèrra la lasciò
}

Il testo segue abbastanza fedelmente la costruzione linguistica dell'italiano che appare nettamente preponderante rispetto agli elementi regionali e dialettali. L'uso lessicale dell'italiano si caratterizza per un impiego di un linguaggio medio con punte di patetismo lirico: foschi, vinse al sé l'onore, scambiata nei color.

Nonostante l'apparente uniformità e regolarità linguistica sono numerosi gli affioramenti del sostrato regionale. Per quanto riguarda l'aspetto fonetico rileviamo: scempiamento dei gruppi consonantici geminati (matina, acorse), passaggio dall'affricata dentale alla fricativa alveolare (ragassina, šia, sensa, gindissio, vissio). Dal punto di vista morfologico l'articolo determinativo davanti all'aggettivo possessivo riferito a nomi di parentela (la sua śia, la mé mama) sono elementi che denotano una maggiore colloquialità pur essendo

\footnotetext{
15 Anesa-Rondi, 1978: 275-276. Per semplificare la lettura e il raffronto fra il testo e il relativo commento riportiamo ciascuna strofa priva della ripetizione degli ultimi due versi. Lo schema testuale riportato in Anesa-Rondi, 1985 è pertanto il seguente:

Ferdinando s'innamóra

di una bèlla, di una bèlla ragassina

che di nóme erà Lisètta

éra nata, éra nata per l'amór

che di nóme erà Lisètta

éra nata, éra nata per l'amór
} 
ugualmente ammessi nello standard. Il dialetto affiora solo all'inizio del primo verso dell'ultima strofa (la mé mama l'à sèmper).

La costruzione sintattica si mantiene semplice ed elementare come nel registro narrativo, anche se notiamo l'uso di una subordinata temporale introdotta da finchè. Riscontriamo inoltre la presenza di un sintagma (il ciel sereno) sintatticamente sciolto dalla frase in cui è inserito.

Siamo in presenza di un uso massiccio dell'italiano, tanto da relegare il dialetto totalmente sullo sfondo, ma con la permanenza di notevoli tratti regionali che connotano localmente il testo.

c) registro puro: ufficiale

Si tratta del registro che maggiormente rispecchia la norma standard della lingua. Proprio per questo possiamo includere nella categoria quei testi, di origine molto più recente rispetto ai casi precedenti, databili all'inizio del Novecento, che in qualche modo subiscono direttamente la pressione di un "linguaggio di massa". I primi a comparire in ordine di tempo sono i canti militari a cui seguono, nel secondo dopoguerra, canti dal sapore popolare ma linguisticamente depurati in vista di una fruizione su tutto il territorio nazionale e non più solo a livello locale.

Riportiamo un breve canto militare che appare particolarmente permeato dallo stile proprio della retorica bellica.

cara la mia Gerania

ti véngo at avvertire

per la Tripolitania

mi toccherà partire.

la guèrra è dichiarata

siam prónti per marciàr

il bravo militare

nessun lo fa tremàr

se incontrerémo i turchi 


\author{
noi li saprém fermare \\ con bómbe e monissióni \\ saprémo bombardare. \\ la guèrra è dichiarata \\ siam prónti per marciàr \\ il bravo militare \\ nessun lo fa tremàr. ${ }^{16}$
}

Il testo padroneggia appieno la lingua italiana secondo la norma. Unica tessera di regionalismo è monissióni, con il passaggio alla fricativa alveolare. Non è presente invece il frequente scempiamento delle geminate che qui appaiono correttamente (avvertire, toccherà, nessun). La struttura sintattica è molto semplice, procede principalmente per giustapposizione ed asindeto, secondo un'assertività tipica del discorso militare. Unica eccezione è l'ipotetica se incontreremo $i$ turchi. Il parlato fa capolino nella frase il bravo militare nessuno lo fa tremàr che presenta una dislocazione a sinistra.

\title{
1.2. Registri di dialetto
}

All'interno delle forme linguistico-testuali in ambito dialettale notiamo un primo registro vicino alla lingua mista italiano-dialetto che realizza una mescolanza imperfetta dei due elementi linguistici. La fusione fra italiano e bergamasco è difficoltosa e tende a configurarsi come un'alternanza fra versi dialettali e versi italiani. Un secondo registro invece presenta una forte coloritura dialettale ma permane una decisa presenza dell'elemento italiano, soprattutto per quanto riguarda i tipi lessicali più evidenti nel discorso. Al terzo livello troviamo testi che fanno un uso diffuso e pieno del codice dialettale. Fra questi spiccano i canti di funzione, che non solo mantengono un'integrità linguistica dovuta alla loro origine arcaica ma esibiscono un uso creativo della lingua più attento alla dimensione del significante che del significato. I canti di funzione si caratterizzano, secondo la teoria etnomusicologica, come creazioni finalizzate all'uso in specifici contesti della quotidianità (come ninne-nanne e canti rituali).

16 Anesa-Rondi, 1978: 303 - 304. 


\title{
a) registro misto: lirico
}

Questo registro è tipico dei canti amorosi dal carattere leggero e scherzoso, a volte malinconico ma mai patetico o drammatico. Sono testi che mescolano liberamente dialetto e lingua italiana, ma le ragioni dell'impasto appaiono meno soggette alle regole e agli schemi che abbiamo verificato per il registro narrativo. I materiali testuali sono trattati con maggior disinvoltura. Inoltre, là dove non ci sono ragioni metriche a regolare la mescolanza dialetto-lingua, l’integrazione fra i due sistemi linguistici appare più difficoltosa e non compiuta. Rispetto al registro narrativo possiede uno statuto di minor letterarietà per avvicinarsi ad un'espressività più diretta ed incisiva.

Riportiamo la prima strofa di Figlia mia ma lü l'è povero.

\author{
figlia mia ma lï l'è pòvero \\ sè lü l'è pòvero non impòrta \\ mé col sach lü con la spòrta \\ ciumbelalì ciumbelalà \\ nè per quèsto lo vòi lascià. ${ }^{17}$
}

Gli elementi lessicali più evidenti di questa strofa sono italiani (figlia mia, povero, importa). Le parole più brevi, spesso parole grammaticali vuote, e per questo in secondo piano, sono in dialetto. In questo caso il testo, lessicalmente con una forte base italiana, riceve una decisa coloritura dialettale. Il fatto che gli elementi grammaticali del testo siano esclusivamente in bergamasco aumenta la percezione di un testo tipicamente vernacolare nonostante le diverse voci estranee al dialetto. In questo caso lingua e dialetto appaiono ben integrati e concorrono unitariamente nel veicolare il messaggio. Attraverso l'uso di parole grammaticali del dialetto il testo riceve una maggiore forza espressiva anche grazie all'immediatezza degli elementi vernacolari.

Troviamo invece altri canti dove l'opposizione fra versi in dialetto e versi in italiano ha una funzione eminentemente espressiva, come nella strofa seguente, che gioca su un

${ }^{17}$ Anesa-Rondi, 1978: 186-188. Traduzione: [Figlia mia ma lui è povero / se lui è povero non importa / io con il sacco lui con la sporta / ... / non per questo lo voglio lasciar]. 
registro ironico, dove $\mathrm{i}$ due versi in dialetto sono incastonati in una coppia di versi in lingua italiana.

$$
\begin{aligned}
& \text { Aspètta ancóra un anno } \\
& \text { epòi te la darò } \\
& \text { ulta 'l fé, pirla 'l fé } \\
& \text { fal sö bé, pòrtel ché } \\
& \text { aspètta ancóra un anno } \\
& \text { epòi te la darò. }
\end{aligned}
$$

In altri casi l'integrazione fra dialetto e lingua può mancare del tutto come nelle seguenti strofe: la prima in bergamasco, la seconda in italiano.

\author{
ol mé murùs si l'è ön vilàn de prima \\ l'à fac de töt per fam inemurà \\ $e$ adès che mé ga ïlìe bé \\ l'm'à ultàt $i$ spale e l'm'à piantàt indré. \\ o vile ingrato che tu fósti stato \\ abbandonare questo miśero cuòr \\ dovévi amarmi volérmi béne \\ farmi godére la felicità ${ }^{19}$.
}

Lo stacco è evidente ed è probabilmente il frutto della saldatura di due canti diversi o della prosecuzione di un più antico canto dialettale secondo uno schema ad innesto. Lo scarto non è solo linguistico ma anche stilistico. La strofa in italiano non risente per nulla di elementi regionali e dialettali. Riscontriamo ancora alcuni termini debitori del patetismo dell'opera lirica (misero cuor, godere la felicita). Addirittura si nota la presenza di

\footnotetext{
18 Anesa-Rondi, 1978: 167 - 168. Traduzione: [.. / . . / volta il fieno, gira il fieno / radunalo bene, portalo qui.]

19 Anesa-Rondi, 1978: 202 - 203. Sono riportate le prime due strofe del canto. Traduzione: [Il mio fidanzato è un mascalzone coi fiocchi/ ha fatto di tutto per farmi innamorare / e adesso che gli volevo bene / $\mathrm{mi}$ ha voltato le spalle e mi ha piantato].
} 
un trapassato remoto (fosti stato) contrariamente alla consuetudine dell'italiano parlato lombardo a evitare l'uso dei tempi storici.

b) registro a predominante: comico-realistico

In questa categoria classifichiamo quei testi a prevalenza dialettale ma che contengono influenze minori della lingua italiana, marginali rispetto all'impianto vernacolare. Tematicamente si tratta di testi a spiccata referenzialità con una vigorosa vena ironica. Il ricorso prevalente al dialetto ha quindi anche lo scopo di rendere più efficace $\mathrm{e}$ graffiante la vis comica sottesa a questi testi che rappresentano una percentuale nutrita all'interno del repertorio.

Riportiamo le due strofe iniziali e quella finale di guardel là che viene adesso:

$$
\begin{aligned}
& \text { guardel là che viene adésso } \\
& \text { che l'gh'à sö le braghe a righe } \\
& \text { sé l'incuntre mé ghe dighe } \\
& \text { che l'istaghe a la sò cà } \\
& \text { che l'istaghe a la sua caśa } \\
& \text { che l'istaghe al suo palasso } \\
& \text { che di sùèn ch'i fa'l paiàsso } \\
& \text { si ghe n'è öna quantità } \\
& \text { (...) } \\
& \text { a l'gnerà la fi d'estate } \\
& \text { che l'marüderà la mèlga } \\
& \text { e di sùuen pié de mèrda } \\
& \text { si l'ghe n'è 'na quantitàa }
\end{aligned}
$$

\footnotetext{
20 Anesa-Rondi, 1978: 220-221. Nella presente riproduzione del testo viene omessa l'onomatopea larilalà ripetuta alla fine di ogni verso. La strofa finale è qui riportata nella variante indicata in coda alla trascrizione della fonte. Traduzione: [Guardalo che viene ora / indossa i pantaloni a righe / se lo incontro gli dico / di stare a casa sua / che stia a casa sua / che stia al suo palazzo / che di giovani che fanno il pagliaccio ce n'è in quantità / ... / verrà la fine dell'estate / maturerà il granoturco / e di giovani pieni di merda / ce ne sono in quantità].
} 
Linguisticamente le strofe hanno un aspetto dialettale. Tuttavia affiorano degli elementi che rimandano all'italiano sia nel senso di una dialettizzazione della lingua nazionale sia nel senso di un maggiore avvicinamento della forma dialettale alla norma grammaticale della lingua standard. Guardel 'guardalo' è un'italianizzazione della voce verbale bergamasca àrdel così come l'articolo determinativo femminile plurale le davanti a braghe rappresenta un influsso della grammatica italiana, dal momento che il bergamasco conosce come articolo determinativo plurale solamente $i$, indipendentemente dal genere $^{21}$. D'altro canto potrebbe anche trattarsi di un tratto presente su influsso dell'area bresciana dove la distinzione fra i due articoli è maggiormente in uso. Ci sono infatti testimonianze orali di le in aree bergamasche marginali, al confine col territorio bresciano, nel quale invece l'articolo femminile è passato dall'antico li a le. Più precisamente il fenomeno è stato riscontrato ${ }^{22}$ sulla riva occidentale del Sebino e parzialmente in Val Calepio. Tali zone sono geograficamente distanti da Parre, paese dove il canto citato è stato raccolto e trascritto, ma le due aree comunicano con facilità grazie al rapido e agevole canale di comunicazione offerto dalla Val Borlezza. Inoltre non va dimenticato che Parre è un paese di forte tradizione pastorizia, i cui abitanti, sovente spostandosi a causa della loro attività, entravano in contatto con realtà dialettali differenti da quelle dell'Alta Valseriana.

Palasso è un tratto di italiano regionale con il classico passaggio dall'affricata dentale alla fricativa alveolare. Contiamo solo cinque termini italiani all'interno delle tre strofe: viene, adesso, righe, quantità, estate.

Presente anche in questo caso un esempio di che polivalente: a l'gnerà la fi d'estate che l'marüderà la mèlga.

\section{d) registro puro: di funzione}

I canti con spiccato tasso funzionale (ninne-nanne, formule rituali, filastrocche, orazioni, invocazioni, rime infantili) costituiscono il gruppo di testi dove il dialetto viene usato con il maggior grado di creatività linguistica. Pressoché assente è l'influenza dell'italiano, mentre è il territorio indiscusso del bergamasco. Si tratta spesso di formule e strofe di probabile origine antica che conservano parole incomprensibili ma di evidente valore

${ }^{21}$ Morfologia del dialetto di Bergamo di Giuliano Bernini, in Sanga, 1987a: 83-118.

22 Bonfadini, 1987b: 397-421 (in particolare pp. 401 e 404). 
onomatopeico. In questo registro il dialetto viene valorizzato nella sua valenza fonosimbolica. L'attenzione è concentrata sugli effetti fonici che è possibile ottenere dalle parole e su associazioni verbali che fanno leva sul pensiero metaforico e simbolico. La tenuta logica della frase e la coerenza testuale passano in secondo piano rispetto alla sapienza dell'uso della lingua in modo evocativo e suggestivo per l'orecchio infantile ed adulto.

Ecco una ninna nanna:

\author{
ninna nanna bèl bambi \\ biànch e rós e risciuli \\ la Madóna l'l'à fasàt \\ San Giuseppe l'l'à adoràt \\ a l'l'à adoràt fón d'ö stansì \\ ciào ciào bèl bambì ${ }^{23}$
}

L'importanza fonico-ritmica nell'economia testuale è evidente dallo schema rimico che inanella tre rime baciate secondo la struttura AABBCC. Non sono presenti elementi dell'italiano, la sintassi è estremamente semplice tanto che al terzo e al quarto verso troviamo delle frasi nucleari senza argomenti o complementi.

\title{
2. Alloglossia e metalinguismo
}

In coda alla sua analisi linguistica sul canto popolare Sanga rileva una scarsa presenza di parole appartenenti a dialetti differenti da quello di Cigole.

La presenza di tratti dialettali estranei al bergamasco è certamente verificabile anche in terra orobica. Come esempio citiamo la seconda strofa di quand'ero piccina, riportata nella raccolta di Dossena.

Grandicella son venuta

All'età di quindic'an

Tengo un figlio che chiama già mamma

23 Anesa-Rondi, 1978: 344. Traduzione: [Ninna nanna bel bambino / bianco rosso e ricciolino / la Madonna l'ha fasciato / San Giuseppe l'ha adorato / l'ha adorato nello stanzino / ciao ciao bel bambino]. 
E non sa chi sia il papa. ${ }^{24}$

La strofa segue la norma grammaticale della lingua nazionale, con gli stilemi tipici della lingua cantata, come il frequente ricorso ad apocopi (son) ed elisioni (quindic'an). Elemento estraneo al sistema linguistico dossenese è l'utilizzo di tengo (riferito a figlio) non nel senso di 'tenere' ma di 'avere', tratto tipico dell'italiano regionale meridionale.

Riportiamo un secondo caso di impiego, ripetuto nel corso del testo, del verbo tenere in senso di ‘possedere'. Il testo proviene da un'area geografica diversa, Parre ${ }^{25}$.

Ió di nóme son cbiamata Teresina

(...)

Tèngo una dòte da fattorèssa

(...)

Tèngo una vigna con un bèl prato

Dopo due occorrenze del verbo tenere viene però usato più correttamente il verbo avere:

\section{(...)}

Ho trénta camicie, vénti sottane,

(...)

Ho un'altra còśa ancor

L'uso di due forme differenti per uno stesso significato, una dall'italiano standard e l'altra dall'italiano regionale o dal dialetto, è un tratto caratteristico dell'ibridazione linguistica dei testi popolari. La tessera alloglotta del verbo tenere in senso di 'avere' mostra quindi una fase più avanzata ${ }^{26}$ di circolazione dei repertori popolari che ormai abbraccia tutta la penisola.

${ }^{24}$ Biella-Zani, 1995: 108-109.

25 Anesa-Rondi, 1978: 268-269.

${ }^{26} \mathrm{Da}$ sottolineare che la raccolta di Dossena è relativamente recente poiché risale al 1995. 
L'elemento alloglotto però non è solo presente in maniera involontaria e nascosta. Può essere un inserimento consapevole e cosciente che nasce intorno a parole ed espressioni linguistiche lontane dalla sensibilità locale e popolare, avvertite quindi come rappresentanti di un mondo altro. È il caso del riuso, con fini parodici, del latino ecclesiastico delle orazioni. Il latino ecclesiastico fino agli anni Sessanta del Novecento fa parte dell'esperienza quotidiana della popolazione italiana, essendo le funzioni religiose celebrate in latino. Tuttavia, nonostante il contatto assiduo con questa lingua, essa rimane ai più un codice sconosciuto o solo debolmente comprensibile.

Distinguiamo due possibili soluzioni espressive: l'incipit della preghiera viene proseguito in dialetto per assonanza, oppure, caso più complesso, il latino attraverso storpiature è avvicinato al dialetto.

Ecco un esempio del primo caso:

\section{Rèchem etèrna}

a laurà s'sa śdèrna

ó lauràt töt estàt

a só töt (e)śdernàt ${ }^{7}$

Il primo verso presenta un latino leggermente rimaneggiato: requiem > rèchem con semplificazione del gruppo [kwie] in [ke]; aeternam > eterna con la caduta di $-m$ postvocalica.

Il latino citato e storpiato in questo canto-filastrocca è quello della preghiera del requiem aeternam (l'eterno riposo), orazione di larga diffusione anche nella religiosità popolare.

Il verso successivo introduce il dialetto rimando con il precedente. Stilisticamente la prosecuzione dialettale che contrappone al sonno eterno enunciato dalla preghiera la stanchezza del lavoro manuale produce un effetto di forte abbassamento espressivo. Attraverso la manipolazione della lingua la psicologia popolare esorcizza la componente luttuosa della quotidianità, prendendo di mira uno dei prodotti culturali imposti dalla morale religiosa. La preghiera del requiem poteva essere vista come simbolo di

27 Anesa-Rondi, 1978: 426-427. Traduzione: [Requiem eternam / a lavorare ci si stanca / ho lavorato tutta estate / e sono tutto stanco.] 
un'esistenza improntata all'austerità dei costumi, che si esplica nell'alto senso del dovere e nel timore reverenziale verso i lati più oscuri dell'esperienza (la morte, il lutto). Ecco invece un caso di interpolazione fra latino e dialetto mediante storpiature:

\author{
Pater noster nostrüos \\ sicut era capelòs, \\ tinga bilinga, \\ foja todesca \\ sed libera nos a malo amen ${ }^{28}$.
}

In questo caso è evidente l'interesse per il puro gioco fonico. Ai due estremi troviamo l'inizio e la fine del Padre Nostro in latino, dopo Pater noster l'aggettivo latino viene ripetuto con una storpiatura. Al verso successivo sembra riprendere il latino (sicut era con caduta di -t postvocalica nel verbo essere alla terza persona) ma due versi nonsense contribuiscono a dare il tono cantilenante di una filastrocca.

Spostandoci dal latino troviamo altri casi di alloglossia che esibiscono con consapevolezza l'elemento linguistico estraneo rendendolo il vero e proprio oggetto del discorso testuale. Classifichiamo quindi questi canti, non numerosi ma con una fortunata diffusione, sotto l'etichetta di metalinguistici, in quanto la riflessione sui fatti di lingua rappresenta l'elemento costitutivo del testo. Sono canti che giocano sulle differenze più evidenti fra le varietà del bergamasco all'orecchio popolare: varianti lessicali oppure fonologiche. Le differenze linguistiche diventano oggetto in canti a sfondo prevalentemente campanilistico dove l'obiettivo comunicativo principale è quello di rinsaldare l'orgoglio della propria appartenenza territoriale.

L'esempio capitale di questa categoria è la nota canzone goliardica notèr de berghem. Riportiamo la prima strofa:

nóter de Bèrghem

de Bèrghem de sùra

à la forchèta à la forchèta

${ }^{28}$ Boninelli, 2010: 100. 


\author{
nòter de Bèrghem \\ de Bèrghem de sùra \\ à la forchèta \\ n'ghe dis ol pirù. ${ }^{29}$
}

Segue poi un ritornello che esibisce una certa mentalità di appartenenza territoriale in chiave possessiva (e che l'è la me càcche 'e qui è la mia casa' ecc...). Il canto, di larghissima diffusione, ironizza sulle differenze linguistiche in materia lessicale fra Bèrghem de sùra e Bèrghem de sóta ${ }^{30}$. La strofa si ripete per tre volte con identico schema. Alla forma di Bèrghem de sóta, caratterizzata da una triplice ripetizione, viene opposta la forma, considerata più autenticamente dialettale dagli abitanti di Bèrghem de sùra, che compare alla fine della strofa come vero e proprio fulmen in clausola. Nelle tre coppie in opposizione, il primo termine (forchèta, finèstra, ürinàre) è un italianismo o comunque una forma più vicina all'italiano. Le differenze lessicali su cui si concentra il canto sono dunque forchèta/pirù, finèstra/balcù, ürinàre/bocàl (vaso da notte). La quarta strofa, la conclusiva, insiste sul termine murùsa 'morosa', ma, con spirito volgare, chiude la strofa con uno scarto: 'n ghe pălpa 'l fiurù ('gli tocchiamo le terga').

Abbiamo quindi preso in considerazione un esempio di metalinguismo popolare in ambito lessicale. Per quanto riguarda quello fonetico riportiamo il canto Hét da Hanga? che è possibile ritrovare in molte raccolte, anche le più antiche ${ }^{31}$.

- Hét da banga?

- Gh'ét la fomna?

- Éla bèla?

- Gh'alaigoh?
- bé ca hò!

- bé ca gh'lò!

- bé ca l'è bèla!

- bé ca la gh'i à!

- cuccurucù ${ }^{32}$

\footnotetext{
${ }^{29}$ Leydi, 1977: 319-320. Traduzione: [Noi di Bergamo / di Bergamo di sopra / la forchetta ... / .. / la forchetta la chiamiamo ol pirii].

${ }^{30}$ Letteralmente, tradotto, 'Bergamo di sopra' e 'Bergamo di sotto', locuzioni che si riferiscono alle due zone del capoluogo orobico: la Città Alta, centro storico di origini romane e medievali, e la più recente città bassa, sviluppatasi sotto il grande impulso dell'industrializzazione otto-novecentesca. Le due parti della città mantengono, almeno nella narrazione popolare e popolaresca, una certa identità e differenza culturale.

${ }^{31}$ Ad esempio in Boninelli, 2010: 53; Bermani-Boninelli, 2001: 144.
} 


\section{- Öt damla a mè?}

Il breve testo, che segue la forma del contrasto, ironizza sulla resa delle sibilanti nell'area della bassa bergamasca. L'indicazione geografica, il toponimo di Zanica ${ }^{33}$, identifica la provenienza dell'interlocutore le cui risposte cominciano sempre con l'avverbio affermativo bè 'sì' a voler marcare l'aspirazione della sibilante. Il carattere ironico prende quindi spunto dall'osservazione di un fatto fonetico tipico delle parlate della pianura bergamasca. La sibilante sorda [s] viene sostituita dall'approssimante laringale sorda $[\mathrm{h}]$ (la cosiddetta "s aspirata") secondo le caratteristiche del sistema fonologico rurale della pianura bergamasca individuato da Sanga ${ }^{34}$.

\section{CONCLUSIONI}

Nel corso del presente articolo è emerso come la descrizione della stratigrafia dei canti appare difficile da appiattire sulla dicotomia lingua/dialetto. La lingua mista identificata da Sanga si presenta piuttosto come un registro intermedio con una differente gradazione di lingua e di dialetto. La mescolanza linguistica possiede diverse ragioni: non solo metriche ma anche funzionali, stilistiche e genetiche. L'uso espressivo del linguaggio in tutte le sue risorse foniche appare un aspetto determinante del corpus folclorico. Non è da trascurare il fatto che questi testi non vengano semplicemente recitati ma cantati e performati. La loro fruizione avviene all'interno di una performance dove determinanti risultano gli aspetti sonori e gestuali dell'azione fisica. Anche la lingua appare dunque modellata da queste istanze. L'uso di differenti registri e codici linguistici permette di portare al massimo grado la valorizzazione fonica della parola in sé e per sé. Dall'altro lato il ricorso a più di un codice linguistico all'interno di un testo permette di impiegare la lingua come un vero e proprio gesto fisico. La parola è spesso esibita con plastica consapevolezza, ad esempio nei casi di alloglossia e metalinguismo. L'elemento estraneo si staglia in rilievo su un fondo omogeneo creando un vero e proprio atto gravido di implicazioni antropologiche e sociali. La consapevolezza delle differenze linguistiche diviene pertanto una potente risorsa nella creazione di un modello

32 Anesa-Rondi, 1975: 424. Traduzione: [- Sei di Zanica - sì che lo sono - hai moglie? - si che ce l'ho - è bella - si che è bella! - ha i gozzi? - si che li ha! - vuoi darla a me? - cuccurucù!].

${ }_{33}$ Paese dell'hinterland posto a sud del capoluogo.

${ }^{34}$ Sanga, 1987a: 19. 
identitario attraverso prodotti culturali come il canto. Allo stesso tempo la coscienza di una realtà sociale bilingue ( $\mathrm{O}$, in certi casi, plurilingue) permette di estremizzare al massimo grado la valenza espressiva dell'espressione verbale fusa con quella musicale.

L'attenzione per il significante piuttosto che per il significato e l'uso diffuso del simbolismo avvicina la lingua di questi testi al concetto di lingua letteraria, nel senso di un codice artificiale lontano dall'uso quotidiano che formalizza ed estremizza alcuni caratteri della lingua parlata. La testualità di numerosi canti inoltre testimonia l'estrema consapevolezza linguistica degli esecutori che si muovono in una situazione di sostanziale diglossia sfruttandola per finalità estetiche e comunicative.

\section{RIFERIMENTI BIBLIOGRAFICI}

Agamennone M., Giannattasio F. (2002) (a cura di), La poesia orale in una prospettiva etnomusicologica, Il poligrafo, Padova.

Agazzi P. (1987), "Il dialetto della Valle Seriana superiore" in Sanga G. (a cura di), Lingua e dialetti di Bergamo e delle valli, vol. 2, Lubrina, Bergamo, pp. 289 - 316.

Ais K. J., Jud J. (1987), Atlante linguistico ed etnografico dell'Italia e della Svizzera meridionale, Unicopli, Milano.

Anesa M., Rondi M. (1978), "Cultura di un paese" in Leydi R. (a cura di), "Cultura di un paese, ricerca a Parre", Mondo Popolare in Lombardia, 6, Silvana d'Arte, Milano, pp. $79-663$.

Bermani C. (1985) (a cura di), "Modestina la va a la morla. Canzoni popolari bergamasche dell'Ottocento. 1 - Il Fondo Carlo Tenca”, in Quaderni dell'Archivio della cultura di base, Sistema Bibliotecario Urbano, Bergamo.

Bermani C., Boninelli M. (2001) (a cura di), “...a veder Garibaldì a rivà. Canzoni popolari bergamasche dell'Ottocento. 2 - La raccolta "Folklore bergamasco" nelle Carte di Paolo Gaffuri (prima parte)", in Quaderni dell'Archivio della cultura di base, Sistema Bibliotecario Urbano, Bergamo. 
Berruto G. (1987), Sociolinguistica dell'italiano contemporaneo, Carocci, Roma.

Biella V., Zani F. (1995), Piamontesi mandim a casa: il canto tradizionale a Dossena, I quaderni della meridiana, Bergamo.

Biella V. (2003), Canti e filastrocche nella media e bassa Valle Seriana. Archivio de "il Popolario" - Archivio "Valter Biella", Bergamo.

Bonfadini G. (1987), "Il dialetto della Val Cavallina e zone adiacenti, saggio di fonetica e fonologia", in Sanga G. (a cura di), Lingua e dialetti di Bergamo e delle valli, vol. 2, Lubrina, Bergamo, pp. 318 - 395.

Bonfadini G. (1987b), "Il dialetto della Val Calepio e Sebino occidentale” in Sanga G. (a cura di), pp. 397 - 421.

Bonfadini G. (1995), "I sistemi consonantici dei dialetti alto-italiani: il caso dell'Alta Val Camonica" in Banfi E. \& al. (a cura di), Italia settentrionale: crocevia di idiomi romanzi, Atti del convegno internazionale di studi (Trento, 21-23 ottobre 1993), Max Niemeyer Verlag, Tübingen, pp. 25-41.

Bonfadini G. (1999), "Profilo dialettale delle valli bresciane", in Boroni C. (a cura di), Itinerari culturali nel bresciano. La montagna., Corbo e Fiore Editori, Venezia, pp. 380 -407 .

Bonfadini G. (2010), lombardi, dialetti in Enciclopedia dell'Italiano, Istituto dell'Enciclopedia Italiana, Roma.

Bonfadini G. (2015), "Il dialetto bresciano alla luce delle ricerche più recenti”, in Piotti M. (a cura di), Dalla scripta all'italiano. Aspetti, momenti, figure di storia linguistica bresciana, Ateneo di Brescia - Morcelliana, Brescia, pp. 263-293.

Boninelli G. M. (2010) (a cura di), ...la sira di serenade. Canzoni popolari bergamasche dell'ottocento. 3 - Le Carte Antonio Tiraboschi. Edizione integrale del Repertorio di canti raccolto nel territorio provinciale. Quaderni dell'Archivio della cultura di base, Sistema Bibliotecario Urbano, Bergamo.

Bravi P., Giannattasio F., Pescatori A. (1994) (a cura di), Il verso cantato. Atti del Seminario di studi, Roma, aprile/gingno 1988, Centro Stampa d'Ateneo - Università degli studi "La Sapienza", Roma.

Cardona G. R. (1976), Introdurione all'etnolinguistica, Il Mulino, Bologna.

Dell'Armellina G. (2008), Ballate popolari europee, BookTime, Milano.

Ettmayer K. (1903), Bergamaskische Alpenmündarten, Leipzig. 
Foppolo B. (1977), "Canti popolari bergamaschi. La Raccolta inedita di Antonio Tiraboschi", in Leydi R. (a cura di), Bergamo e il suo territorio, Mondo popolare in Lombardia 1. Milano, Silvana: 425 - 515.

Giannattasio F. (2005), "Dal parlato al cantato", in Nattiez J. J., (a cura di), Enciclopedia della musica, vol. V, Einaudi, Torino.

Giusti M. E. (2006), “La Canzone narrativa fra poesia e canto”, in Priore R. (a cura di), La canzone narrativa. Volume terzo. Documenti di canto e poesia popolare raccolti nella Valdarno superiore. Comune di Terranuova di Bracciolini, pp. 7 - 17.

Halliday M. A. K. (1983), Il linguaggio come semiotica sociale. Un'interpretazione sociale del linguaggio e del significato, Zanichelli, Bologna.

Leydi R. (1972) (a cura di), Le trasformazioni socio-economiche e la cultura tradizionale in Lombardia, Regione Lombardia, Milano.

Leydi R. (1973), I canti popolari italiani: 120 testi e musiche scelti e annotati, Mondadori, Milano.

Leydi R. (1977), "Per la conoscenza della musica popolare nel territorio di Bergamo", in Id (a cura di), Bergamo e il suo territorio, Mondo popolare in Lombardia 1. Milano, pp. $261-342$.

Leydi R. (1990), "Sentite buona gente. La ballata e la canzone narrativa", in Id (a cura di), Le tradizioni popolari in Italia. Canti e musiche popolari, Electa, Milano.

Meazza R., Scaldaferri N. (2008) (a cura di), Patrimoni sonori della Lombardia. Le ricerche di Etnografia e Storia Sociale. Squilibri, Roma.

Neziri Z. U., Scaldaferri N. (2016), "From the Archive to the Field: New Research on Albanian Epic Songs", in Elmer D. F., McMurray P. (a cura di), Issue 14: Singers and Tales in the 21st Century; The Legacies of Milman Parry and Albert Lord, Classics@, Harvard.

Ong W. J. (1987), Oralità e scrittura, Il Mulino, Bologna.

Saffiotti T. (2008), Ballate popolari italiane, BookTime, Milano.

Sanga G. (1979), Il linguaggio del canto popolare, MEDI Sviluppo - Giunti/Marzocco, Milano.

Sanga G. (1984), Dialettologia Lombarda, Aurora, Pavia.

Sanga G. (1987a) (a cura di), Lingua e dialetti di Bergamo e delle valli, vol.1, Bergamo, Lubrina, Bergamo. 
Mattia Sonzogni, La mescolanza linguistica come forma di comunicazione espressiva: il caso del canto popolare bergamasco

Sanga G. (1987b) (a cura di), Lingua e dialetti di Bergamo e delle valli, vol .2, i dialetti delle valli, Lubrina, Bergamo.

Sanga G. (1987c) (a cura di), Lingua e dialetti di Bergamo e delle valli, vol .3, l'italiano regionale bergamasco, Lubrina, Bergamo.

Saussy H. (2016), The Etnography of rhythm. Orality and his Technologies, Fordham University Press, New York. 